\title{
ION BEAM DYNAMICS IN POLYHARMONIC FIELD OF LINAC
}

\author{
E.S. Masunov $^{\#}$, N.E. Vinogradov, MEPhI, 115409, Moscow, Russia
}

\section{Abstract}

This paper describes the problem of ion RF focusing in the axisymmetric RF field involving one synchronous and several nonsynchronous harmonics. The influence of nonsynchronous harmonics on ion beam dynamics is studied by means of 3D effective potential, which is obtained for smooth approximation. The motion equation is presented in Hamilton's form. The possibility of simultaneous transverse focusing and phase stability for accelerating ion beam in the RF field without the synchronous harmonic is shown.

\section{INTRODUCTION}

It is known, that in order to achieve simultaneous transverse focusing and phase stability for the accelerating ion beam in linac it is necessary to use external focusing elements or to apply a special configuration of the RF field. The second way is more effective for low energy ion RF accelerators . In papers [1-3] it was shown that the RF system with several traveling waves, one being synchronous with the beam may provide simultaneously acceleration and focusing of ions. The systems with infinite numbers of harmonics were investigated numerically [4]. Besides, it was shown that the required effect can be achieved in the undulator accelerators, where the synchronous harmonic is absent [5]. In this paper the method of analysis of 3-D beam dynamics in the polyharmonic RF field of linac is suggested. For this purpose the averaging method is used. In such approach the motion equation has the Hamilton's form. It allows us to take into account the relationship between transverse and longitudinal oscillations. The shape of the effective potential obtained describes the 3D motion of the particle completely.

\section{MOTION EQUATION}

The RF field in the periodical structure can be presented in the form

$$
\begin{aligned}
& E_{z}=\sum_{n=0}^{\infty} E_{n}{ }^{z} \cos \left(h_{n} z\right) \cos (\omega t), \\
& E_{r}=\sum_{n=0}^{\infty} E_{n}{ }^{r} \sin \left(h_{n} z\right) \cos (\omega t),
\end{aligned}
$$

where $\quad E_{n}{ }^{2}=E_{n} I_{0}\left(h_{n} r\right), \quad E_{n}{ }^{r}=E_{n} I_{1}\left(h_{n} r\right)$, $h_{n}=h_{0}+2 \pi n / D, h_{0} D$--a phase advance per period

\# E-mail:masunov@dinus.mephi.ru of structure $D$. Trajectories of particles in the field (1) may be expressed by the summation of two different types of motion $\boldsymbol{r}_{s}$ and $\tilde{\boldsymbol{r}}$, which are caused by slowly varying field and the rapidly oscillating one respectively. By means of averaging method the motion equation for slow component can be obtained in the form

$$
\frac{d^{2} \boldsymbol{R}}{d \tau^{2}}=-\frac{\partial}{\partial \boldsymbol{R}} U_{e f f}
$$

where $U_{\text {eff }}=U_{0}+U_{1}+U_{2}+U_{3}$ is the effective potential function:

$$
\begin{gathered}
U_{0}=\frac{1}{2} e_{s}^{z} \sin \psi \\
U_{1}=\frac{1}{16} \sum_{n \neq s} \frac{\boldsymbol{e}_{n}{ }^{2}}{\Delta^{-}{ }^{2}}+\frac{1}{16} \sum_{n} \frac{\boldsymbol{e}_{n}{ }^{2}}{\Delta^{+}{ }^{2}} \\
U_{2}=\frac{1}{16} \sum_{\substack{n \neq s \\
n+p=2 s}} \frac{e_{n}{ }^{z} e_{p}{ }^{z}-e_{n}{ }^{r} e_{p}{ }^{r}}{\Delta^{-}{ }^{2}} \cos (2 \psi) \\
U_{3}=\frac{1}{16} \sum_{p \neq s} \frac{\boldsymbol{e}_{n} \boldsymbol{e}_{p}}{\Delta_{s, p}^{-}{ }^{2}} \cos (2 \psi),\left|h_{p}-h_{n}\right|=2 h_{s} .
\end{gathered}
$$

Here $\quad e_{n}=e h_{s} E_{n} / m \omega^{2}, \quad e_{n}^{z, r}=e_{n} I_{0,1}\left(h_{n} r\right)$, $\boldsymbol{R}=2 \pi \boldsymbol{r}_{s} / \lambda \beta_{s}, \quad \tau=\omega t, \quad \Delta_{s, n}{ }^{ \pm}=\left(h_{n} \pm h_{s}\right) / h_{s}$, $\psi$ and $\beta_{s}$--are phase and velocity of synchronous particle and $\mathrm{s}$ is synchronous harmonic's number. In the following the variable $\chi=z-z_{s}$ and $r$ will be used as a longitudinal and transverse variable respectively. The effective potential function $U_{\text {eff }}$ describes particle dynamics in the RF field in smooth approximation completely. The result is that the equation (2) is presented in Hamilton's form. The function $U_{\text {eff }}$ connects longitudinal and transverse motions of ion and allows us to investigate their influence on each other. The first summand $U_{0}$ describes the interaction between particle and synchronous wave, the second one $U_{l}$ defines the focusing action of nonsynchronous harmonics. The functions $U_{2,3}$ are mixed terms, which are describing the influence of nonsynchronous waves both on transverse and longitudinal dynamics. Simultaneously radial and phase stability takes place when the function $U_{\text {eff }}$ has an absolute minimum. This is possible when some conditions on amplitudes and phase of harmonic are satisfied. 


\section{SYNCHRONOUS AND ONE NONSYNCHRONOUS HARMONICS}

For two harmonics s and $\mathrm{n}$ the item $U_{2}=0$. Let's define the frequencies of radial and phase oscillations as $\omega_{r}(r)=\frac{1}{r} \frac{\partial U_{e f f}}{\partial r} ; \quad \omega_{z}(\chi)=\frac{1}{\chi} \frac{\partial U_{e f f}}{\partial \chi}$.

The

conditions of transverse and longitudinal stability are

$$
\omega_{r}{ }^{2}>0, \omega_{z}{ }^{2}>0
$$

Then in the linear approximation $\omega_{r}^{2}(0)=\omega_{r 0}{ }^{2}+\omega_{r l}{ }^{2} \cos (2 \psi)$, $\omega_{z}{ }^{2}(0)=\omega_{z 0}{ }^{2}+\omega_{z 1}{ }^{2} \cos (2 \psi)$, where $\omega_{r, z 1}$ are defined by term $U_{3}$ :

$$
\begin{aligned}
& \omega_{r 0}{ }^{2}=-\frac{1}{4} e_{s} \sin \psi+\frac{3}{32} \frac{e_{s}^{2}}{\Delta_{s, s}^{+}{ }^{2}}+ \\
& +\frac{3}{32} e_{n}^{2}\left(\frac{1}{\Delta_{s, n}^{+}{ }^{2}}+\frac{1}{\Delta_{s, n}^{-}}\right)\left(\frac{h_{n}}{h_{s}}\right)^{2} \\
& \omega_{r 1}^{2}=\frac{1}{16} \frac{e_{s} e_{n}}{\Delta_{s, n}^{-}} \frac{h_{n}^{2}+h_{s}^{2}+h_{n} h_{s}}{h_{s}^{2}} \\
& \omega_{z 0}{ }^{2}=\frac{1}{2} e_{s} \sin \psi ; \omega_{z 1}{ }^{2}=\frac{1}{2} \frac{e_{s} e_{n}}{\Delta_{s, n}^{-}}
\end{aligned}
$$

It can be seen from (4), (5) that the larger the ratio $e_{n} / e_{s}$, the more effective transverse focusing is. Besides, this focusing is stronger when $s<n$, i.e. the acceleration wave is faster than the focusing one. The simple analysis shows that for the case $e_{n} / e_{s} \gg 1$ impact of the term $U_{3}$ is unessential and $\omega_{r, z l}$ can be neglected. In the following we will assume $e_{n}=e_{\max }$, $e_{s}=k e_{\max }$, where $k<1$ and $e_{\max }$ is defined by the breakdown voltage. The critical value of $k$ can be found from (4). The overlapping of the phase stability buckets of neighboring harmonics is undesirable. Thus, the parameter $e_{\max }$ is also bounded above:

$$
\sqrt{2} e_{\max }^{1 / 2}\left(1+k^{1 / 2} v_{s} / v_{n}\right)<\left|\Delta_{n, s}{ }^{-}\right|
$$

The function $U_{\text {eff }}$ allows us to take into account the relationship between radial and phase oscillations accurately. In the simplest approach [7], considering the longitudinal oscillations to be in the form $\chi=\Phi \sin \left(\omega_{z} \tau\right)$, we can obtain from (2) the equation of the transverse motion as $d^{2} r / d u^{2}+\pi^{2}(a+2 q \sin 2 \pi u) r=0 \quad$ with $a=\left[2 \omega_{r}(0) / \omega_{z}(0)\right]^{2}, q=\Phi \operatorname{ctg} \psi ; \Phi$ is the amplitude of longitudinal oscillations. Then the condition of resonance is

$$
2 \omega_{r}(0) / \omega_{z}(0)=l, l=1,2,3, \ldots
$$

The zone of instability corresponds to each $l$ in (7). The conditions (4), (6), (7) formulate the restrictions on amplitudes of harmonics. The diagram of stability including only conditions (4) and (7) is shown in Figure 1. In order to investigate the dependence of transverse frequency and/or transverse emittance on harmonic's numbers one can fix the parameters $e_{\max }, k, \psi$ (i.e. fix the $\omega_{z}$ and/or the longitudinal emitance) and calculate the value of $\omega_{r}$ for different $s, n$ and $h_{0}$. This analysis was done for the proton acceleration system with $E_{\text {max }}=100 \mathrm{kV} / \mathrm{cm}, \mathrm{k}=0.15, \quad \psi=\pi / 4, \quad \beta_{s}=0.015$, $\lambda=200 \mathrm{~cm}$. The most effective transverse focusing is provided by the system with $s=2, n=3$ for $h_{0}=0$ and $s=0, n=1$ for $h_{0}=\pi / D$. Besides, the radial focusing is more effective for the systems with $s<n$.

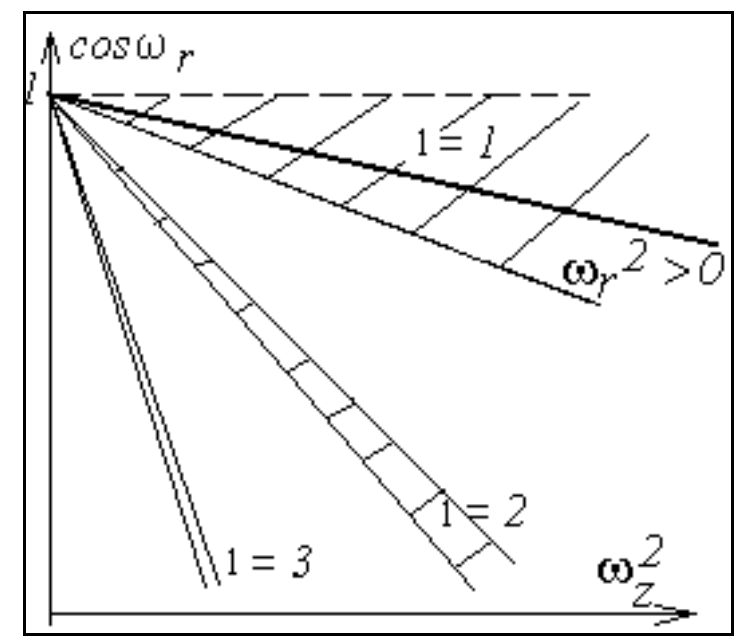

Figure 1: The transverse stability and the zones of parametric resonance

\section{SYNCHRONOUS AND TWO NONSYNCHRONOUS HARMONICS}

Let's add one more nonsynchronous harmonic with number $\mathrm{p}$ to the RF field discussed above. In this case summands $U_{2}$ and $U_{3}$ can be not equal to 0 . These terms render acceleration and defocusing influence on particle motion. Here the items $U_{2,3}$ are of the same order as $U_{1}$ so it is necessary to take them into account. The analysis of influence of the summands $U_{2,3}$ shows that these terms deform the shape of $U_{\text {eff }}$ significantly. So the phase capture is reduced considerably. At the same time, the contribution of the items $U_{2,3}$ into the acceleration gradient can exceed the contribution of $U_{0}$ in several times. It means, that such system may turn out 
to be effective for acceleration of the bunched beam. The transverse emittance and/or $\omega_{r}$ is determined by the ratio between terms $U_{1}$ and $U_{2,3}$. Figure 2 shows $\omega_{r}(r)$ for different combinations $\mathrm{s}, \mathrm{n}, \mathrm{p}$. The parameters of the system are the same as those discussed above; $\mathrm{k}=0.3$, $e_{p}=e_{\max }$. The most effective transverse focusing is provided by the system with $s=2, n=1, p=3$ and $h_{0}=0$.

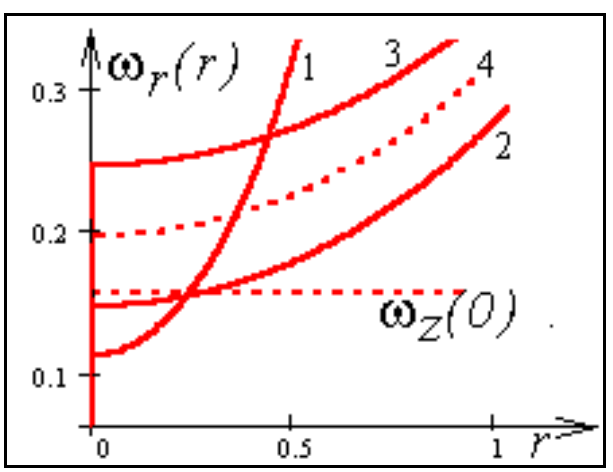

Figure 2: The dependence of transverse frequency on the radius

$1-\{\mathrm{s}=0, \mathrm{n}=1, \mathrm{p}=2\} ; 2-\{\mathrm{s}=1, \mathrm{n}=0, \mathrm{p}=2\} ; 3-\{\mathrm{s}=2, \mathrm{n}=1$, $\mathrm{p}=3\} ; h_{0}=\pi / D .4-\{\mathrm{s}=2, \mathrm{n}=1, \mathrm{p}=3\} ; h_{0}=0$.

\section{TWO NONSYNCHRONOUS HARMONICS}

The conclusion about the influence of nonsynchronous waves on longitudinal dynamics of the beam becomes even more convincing if in the RF system, discussed in the previous chapter the synchronous harmonic is absent.

Table 1: The comparison of different acceleration systems

\begin{tabular}{|ll|l|l|l|}
\hline & & $e_{s, n} \neq 0$ & $e_{s, n, p} \neq 0$ & $e_{n, p} \neq 0$ \\
\hline \multirow{4}{1}{} & $\mathrm{s}=1$, & $\omega_{r}=0.13$ & $\omega_{r}=0.10$ & $\omega_{r}=0.16$ \\
& $\mathrm{n}=2$, & $\omega_{z}=0.17$ & $\omega_{z}=0.24$ & $\omega_{z}=0.17$ \\
& $\mathrm{p}=0$, & $\mathrm{T}=0.75$ & $\mathrm{~T}=1.19$ & $\mathrm{~T}=1.1$ \\
\hline \multirow{2}{*}{$\mathbf{2}$} & $\mathrm{s}=2$, & $\omega_{r}=0.18$ & $\omega_{r}=0.16$ & $\omega_{r}=0.19$ \\
& $\mathrm{n}=3$, & $\omega_{z}=0.17$ & $\omega_{z}=0.29$ & $\omega_{z}=0.23$ \\
& $\mathrm{p}=1$ & $\mathrm{~T}=0.75$ & $\mathrm{~T}=2.7$ & $\mathrm{~T}=1.96$ \\
\hline
\end{tabular}

$T \equiv d W_{s} / d z_{s}, \mathrm{MeV} / \mathrm{m} ; 1: h_{0}=\pi / D ; 2: h_{0}=0$

In this case the influence of focusing term $U_{1}$ and accelerating ones $U_{2,3}$ may turn out to be sufficient to create 3D potential well in which radial and phase oscillations are stable. Besides, the conditions similar to (6) and (7) can be obtained. The comparison of characteristics of different acceleration systems are shown in Table 1. The parameters of the system are the same as those discussed above, $\psi=\pi / 3$.
One can see that acceleration and transverse focusing in the RF field without synchronous harmonic is possible. Moreover, such system may be more effective than the standard one with synchronous and one nonsynchronous waves.

\section{COMPUTER SIMULATION}

In order to test all the results obtained the computer simulation was done. The motion equation in the field (1) was solved numerically for all the acceleration systems discussed. It was shown that the averaging method allows us to describe the behavior of the beam correctly. The range of velocities where the investigation of the beam dynamics by means of effective potential function $U_{\text {eff }}$ is completely valid is bounded below. For instance, in the case of protons $\beta_{s}>0.005$.

\section{CONCLUSION}

The method of analysis of the ion beam's dynamics in the polyharmonic RF field is suggested. The RF system with synchronous and one nonsynchronous harmonics is investigated completely. The conditions of stability are obtained. The features of the acceleration system with two nonsynchronous waves are shown. The possibility of simultaneous longitudinal and transverse focusing of the beam in the field without synchronous harmonic is exhibited. The comparison of the acceleration system discussed is done. All results are tested by computer simulation of beam dynamics in the RF field.

\section{REFERENCES}

[1] V.S. Tkalich, Zh. Ex. and Theor. Fiz. Vol. 32, p. 625, 1957.

[2] V.K. Baev, N.G. Gavrilov, S.A. Minaev and A.V. Shalnov, Zh. Tekn. Fiz. Vol. 53, p. 1287, 1983.

[3] V.D. Danilov, A.A. Iliin, Y.K. Batygin, Proc. 3 Europ. Part. Accel. Conf., Berlin, vol.1, p. 569, 1992.

[4] H.Okamoto, Nucl. Instr.and Meth. in.Phys. Res. A 284, p233, 1989.

[5] E.S. Masunov, Proc. of the 18 Internation. Linac. Confer., Geneva, Switzerland, CERN 96-07, 1996, vol. 2, p. 487-489.

[6] E.S. Masunov, N.E. Vinogradov, 15 Internation. Linac Workshop, Alushta, Krim, 1997, vol 1, p.184-186.

[7] I.M. Kapchinsky. Theory of Res.Linear Accelerator, Harwood, 1985. 